\title{
Reconstructing the invasion and the demographic history of the yellow-legged hornet, Vespa velutina, in Europe
}

\author{
M. Arca - F. Mougel • T. Guillemaud - S. Dupas • Q. Rome - A. Perrard \\ F. Muller - A. Fossoud $\cdot$ C. Capdevielle-Dulac $\cdot$ M. Torres-Leguizamon • \\ X. X. Chen · J. L. Tan • C. Jung • C. Villemant - G. Arnold - J.-F. Silvain
}

\begin{abstract}
The yellow-legged hornet, Vespa velutina, was accidentally introduced from Southeast Asia and then invaded France and Korea over the last 10 years. Since its introduction, its predation on honeybee colonies has rapidly become an economic problem in invaded countries. Using mitochondrial cytochrome C oxidase and 22 nuclear microsatellite loci, we showed that native hornet populations were well differentiated and highly diverse. In contrast, introduced populations from France and Korea suffered a genetic bottleneck, which did not prevent their rapid geographic
\end{abstract}

Data accessibility DNA sequences: Genbank accessions JQ780449 JQ780462.

Electronic supplementary material The online version of this article (doi:10.1007/s10530 0150880 9) contains supple mentary material, which is available to authorized users.

M. Arca $(\bowtie) \cdot$ S. Dupas · A. Fossoud .

C. Capdevielle Dulac · M. Torres Leguizamon •

J. F. Silvain

IRD, UMR EGCE (Evolution, Génome, Comportement et Ecologie) Univ. Paris Sud CNRS IRD, Univ. Paris

Saclay, Gif sur Yvette, France

e mail: mariangela.arca@gmail.com

J. F. Silvain

e mail: Jean Francois.Silvain@legs.cnrs gif.fr

M. Arca · F. Mougel - S. Dupas · C. Capdevielle

Dulac . M. Torres Leguizamon - G. Arnold ·

J. F. Silvain

Université Paris Sud 11, 91405 Orsay Cedex, France expansion. Analysis of the genetic data indicates that French and Korean populations likely arose from two independent introduction events. The most probable source population is from an area between the Chinese provinces of Zhejiang and Jiangsu. This invasion route is in agreement with knowledge on trade and historical records. By studying colonies of $V$. velutina, we demonstrated its polyandry, which is very rare among Vespidae. This mating behavior could have favored the success of this Asian hornet in Europe and Korea. Combined, the population and colony results suggest that very few or possibly only one single multi-mated female gave rise to the invasion.

Keywords Yellow-legged hornet - Vespa velutina . Invasive species - Approximate Bayesian computation

\author{
M. Arca · F. Mougel - G. Arnold \\ CNRS, UMR EGCE (Evolution, Génome, Comportement \\ et Ecologie) Univ. Paris Sud CNRS IRD, Univ. Paris \\ Saclay, Gif sur Yvette, France \\ T. Guillemaud \\ INRA, Univ. Nice Sophia Antipolis, CNRS, UMR \\ 13557254 Institut Sophia Agrobiotech, 400 Route des \\ Chappes, BP 167, 06900 Sophia Antipolis, France \\ Q. Rome · A. Perrard · F. Muller · C. Villemant \\ UMR7205, CP50, Muséum National d'Histoire Naturelle, \\ 45 rue Buffon, 75005 Paris, France
}




\section{Introduction}

The increases of global trade and human mobility over the last century have resulted in unprecedented numbers of invasions by non-native species all over the world. These biological invasions have severe impacts on agriculture and natural resources (Mack et al. 2000). Social insects are among the most successful invasive groups (Moller 1996). Their invasive success has been attributed to life-history traits that facilitate their introduction and expansion into new habitats (Holway et al. 2002). Sociality is generally associated with behavioral flexibility, which may contribute to the invasive success of some social species. For example, introductions of ants, which are among the most widespread and damaging alien species, are often accompanied by alterations in social behavior and colony structure that contribute to their success (Tsutsui et al. 2001; Suarez and Tsutsui 2008).

Another key to the invasive success of social insects is the ability of a single queen to found a large colony and to produce many reproductive females that then offer a large dispersal capacity (Moller 1996; Chapman and Bourke 2001; Schmid-Hempel et al. 2007; Mikheyev et al. 2009).

Association with human activities such as pollinisation is another element that makes social insects successful invaders because of deliberate displacement of large samples of individuals and often repetitive events of introduction. This association is particularly relevant for bees (Bombus and Apis spp.), which have been introduced to many new areas for economic reasons (Moller 1996; Ings et al. 2006).

Among social hymenoptera, Vespa species are not commonly considered invasive. Several species have been introduced to areas beyond their native ranges,

X. X. Chen

State Key Laboratory of Rice Biology and Ministry of Agriculture Key Laboratory of Molecular Biology of Crop Pathogens and Insects, Institute of Insect Sciences,

Zhejiang University, Hangzhou 310029, China

J. L. Tan

School of Life Sciences, Northwest University, 229 Taibai North Road, Xi' an 710069, Shaanxi, China

C. Jung

School of Bioresource Sciences, Andong National University, Andong 760 749, Korea but most failed to become established (Beggs et al. 2011; Villemant et al. 2011). One Vespidae that has made rapid and high-profile invasions is the yellowlegged hornet, Vespa velutina, recently introduced and established in both France and Korea. This was the first successful invasion of an exotic vespid into Europe (Villemant et al. 2011) and it was noticeable for its velocity. Vespa velutina naturally occurs in Asia, from Afghanistan to eastern China, Indo-China and Indonesia (Carpenter and Kojima 1997), where it is known as an active predator of honeybees (Abrol 1994; Tan et al. 2007). The first field record of the yellow-legged hornet in France was obtained in the Lot-et-Garonne département in 2005, although, according to local testimonies, it was likely present as early as 2004 (Villemant et al. 2011). Following its introduction, the hornet spread rapidly across southwest France. The invaded area had reached about 190,000 $\mathrm{km}^{2}$ by 2010 (Villemant et al. 2011; Fig. 1) and 360,000 by 2012 (Rome et al. 2013). The hornet was also reported in northern regions of Spain and in northern Portugal. One male was also recorded in Belgium in 2011 (Rome et al. 2013). Comparisons of the climatic niches between the native and invasive range revealed that many countries of Western Europe have climatic conditions suitable for $V$. velutina and, therefore, a high probability of being invaded (Villemant et al. 2011). The first hornet observation in France was made by a French bonsai producer who regularly imported pots from Chinese coastal areas close to Shanghai (Villemant et al. 2011). It has thus been suggested that the first introduction of the Asian yellow-legged hornet into France was associated with an importation of horticultural pots carried on cargo boats from these Chinese provinces (Villemant et al. 2006). It is well known that social Vespidae are transported passively by man, as fertilized queens seek sheltered locations in which to undergo diapause. Such shelters are often found in human goods, which may then be transported to new locations with the queens as passengers (Beggs et al. 2011). A full nest could not voyage in this way as, under the same conditions, it would be detected and destroyed.

Little is known about the $V$. velutina introduction to Korea. The hornet was found for the first time on the Bongrae Mountain, Yeong-do districts, near Busan, in 2003. Vespa velutina appears to have spread much more slowly in Korea (Choi et al. 2012) than in France 


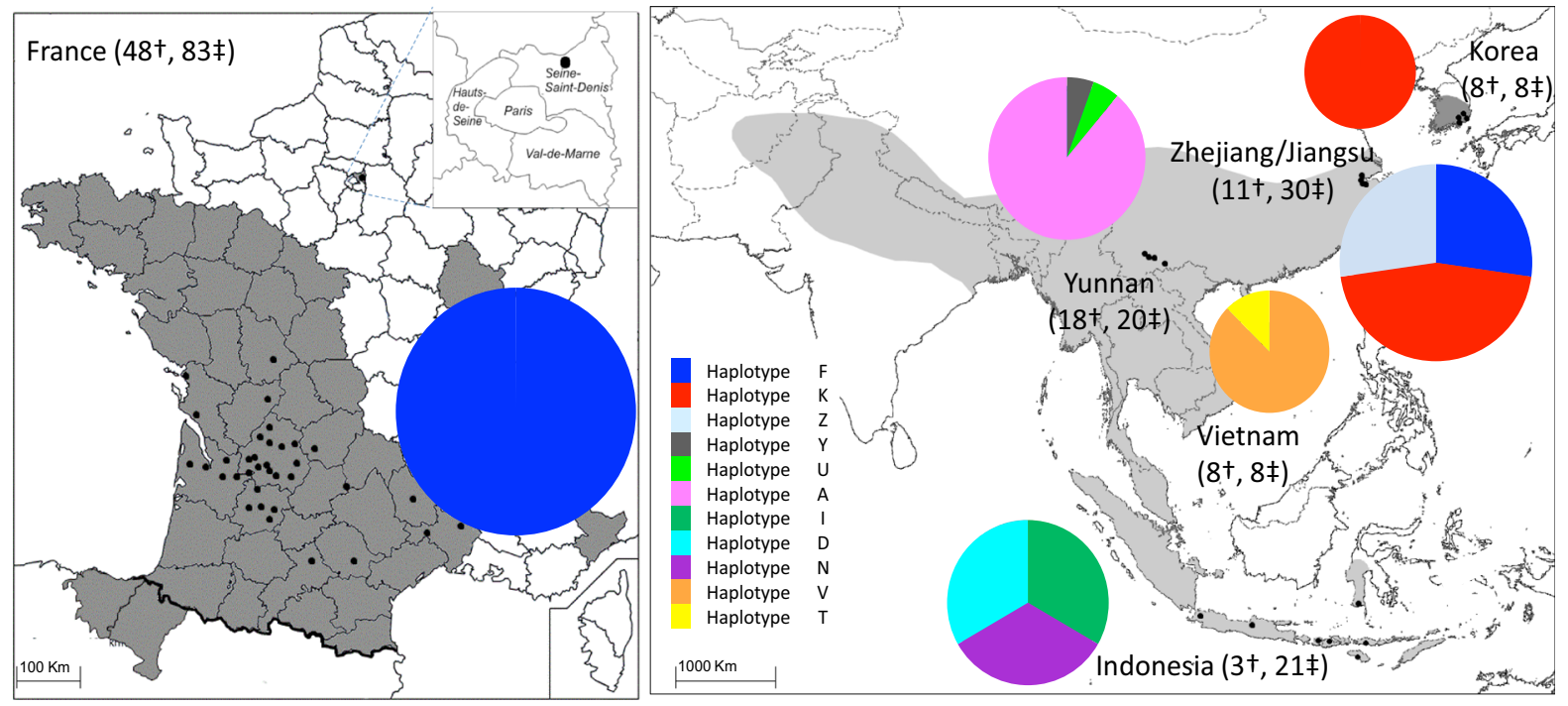

Fig. 1 Map of the six regions sampled for the yellow legged hornet Vespa velutina. Each point on the map represents a sampled locality. Grey areas indicate the known distribution range of $V$. velutina in 2010. The pie charts display haplotype frequency for the mitochondrial COI marker in each sampled region and their size is proportional to the sampling effort. The

(Rome et al. 2013). The reason for this might be that the invasive hornet must contend with six other Vespa species in Korea, whereas only one, V. crabro, is present in France (Villemant et al. 2011; Choi et al. 2012). Vespa velutina is currently abundant in the southern part of Korea and has spread over one-third of the country as a whole (Choi et al. 2013).

Like many other invasive species, V. velutina has never caused significant economic problems in its native range, where it is likely that many biotic factors (e.g., predation and competition) control its population levels. To our knowledge, such factors are absent from the area of France where this species was introduced. Multiplication of the species has also probably been greatly enhanced by the abundance of the European honeybee Apis mellifera throughout the whole of Europe (Perrard et al. 2009; Monceau et al. 2013). Apis mellifera is probably the hornet's main prey in Europe because, in contrast with the Asian species Apis cerana (Villemant 2008; Ken et al. 2005), this honeybee has no effective defensive behavior to fight against its new predator (Rortais et al. 2010; Monceau et al. 2013). Yellow-legged hornets feed on a diversity of insects and may thus also have a significant impact on other native arthropods (Villemant et al. 2011). The haplotype names are associated with a color code as indicated in the legend. Dagger number of samples used for mitochondrial analysis; double dagger number of samples for microsatellite analysis. All samples are listed in table S1 in which samples used for mtDNA characterization are in bold

impact of $V$. velutina on insect diversity is difficult to assess, while the damage to French apiaries is obvious: high losses due to deaths of colonies and to reduction of honey production has led some beekeepers to cease apiculture activities (Villemant et al. 2011). In addition to damages caused to apiaries, V. velutina is alarming to human populations due to its huge nest and population size.

The objective of this study is to genetically trace the history of the introduction of $V$. velutina in France and Korea in order to (1) identify the geographic origin of the introduced propagules, (2) estimate the number of founders responsible for the establishment of the invasive population, and (3) improve knowledge about the life history traits of $V$. velutina that may have facilitated its demographic success in the invaded areas.

\section{Materials and methods}

Sample collection and DNA isolation

For the population study, a total of 170 samples of $V$. velutina were obtained from six geographic areas 
(Fig. 1): France (FRA, $n=83)$, Korea $(K O R, n=8)$ (invaded areas), Vietnam (VIE, $\mathrm{n}=8$ ), Indonesia (IND, $\mathrm{n}=21$ ), Yunnan (YUN, $\mathrm{n}=20$ ) and Zhejiang/ Jiangsu provinces (ZHE/JIA, $\mathrm{n}=30$ ) (native areas) (Table S1). Females were collected in front of hornet nests, in front of beehives or using beekeeper's traps, containing a food attractant. When a sweeping net was used in front of the nests, only one female per nest was analyzed. For the hornets captured away from their nests (in beekeeper's traps or in front of beehives), only one sample per locality was genotyped from localities situated at least $3 \mathrm{~km}$ away from one another in order to limit the chance of sampling individuals from the same colony. Because a restricted number of individuals were sampled from each region, individuals were treated as representative samples from each country studied. Consequently, the term 'population' refers to the total number of individuals from each sampled country (or province in the case of China). Mean distance between samples was $99 \mathrm{~km}$ in France, $53 \mathrm{~km}$ in Korea, $3 \mathrm{~km}$ in Vietnam, $591 \mathrm{~km}$ in Indonesia, $177 \mathrm{~km}$ in Yunnan and $60 \mathrm{~km}$ in Zhejiang/ Jiangsu.

In addition to the isolated hornets collected in the field for population genetic studies, we analyzed samples of $V$. velutina from 10 different colonies in order to determine the number of progenitors (reproductive females and their mates). Colony samples were collected in three areas of France: Dordogne, Gironde and Ile-de-France. A total of 396 females were DNA-genotyped. DNA was extracted from pupae or adults (thorax or legs) using the 'DNeasy tissue Kit' (Qiagen).

Mitochondrial DNA sequencing and analysis

Mt DNA of 48 individuals among the $83 \mathrm{~V}$. velutina from France and 48 among the 87 samples from Asia (Indonesia, Vietnam, China and Korea) was amplified using universal primer sequences HCO-2198 and LCO-1490 to yield a 658 bp fragment of the mitochondrial gene cytochrome C oxidase subunit I (COI) (Folmer et al. 1994). Samples were chosen to cover the widest geographic range as possible (Table S1).

Polymerase Chain Reaction (PCR) amplifications were performed according to the standard PCR reaction protocol used at the Canadian Centre for DNA Barcoding (Hajibabaei et al. 2005). PCR products were then verified on a $2 \%$ agarose gel. Purified PCR fragments obtained from the $\mathrm{HCO} / \mathrm{LCO}$ primer pair were sequenced in both directions. Both strands of DNA were aligned manually using BIOEDIT 7.0.5.3 (Hall 1999). Edited sequences of each haplotype were deposited in GenBank (accession numbers: JQ780449 JQ780462). Haplotype and nucleotide diversity (Nei 1987) were calculated using DnaSP 4.10.9 (Rozas et al. 2003). A haplotype parsimony network was reconstructed using TCS 1.21 (Clement et al. 2000), as described by Templeton et al. (1992), with a probability cut-off set at $95 \%$.

Microsatellite amplification and analysis

Seven microsatellite loci previously developed for other Vespidae were used for V. velutina (Hasegawa and Takahashi 2002, Daly et al. 2002). Fifteen additional loci isolated from $V$. velutina were used in the present study (Arca et al. 2011). All 170 hornets were genotyped at 22 microsatellite loci (listed in Table S1, Supplementary materials).

PCR amplifications were performed as described in Arca et al. 2011. PCR products were analyzed in an ABI Prism ${ }^{\circledR} 3100$ Genetic Analyzer (Applied Biosystems). Alleles were scored with GeneMapper v.4.1 (Applied Biosystems), and each allele-call was checked manually. Due to amplification problems with heterologous loci (List2003, VMA8, VMA6, LIST2018B and LIST2004B), we could not obtain complete genotypes for 30 of the Chinese, the 8 Korean and 15 of the Indonesian specimens (Table $\mathrm{S} 1)$. As full genotype was obtained for the remaining samples, all 22 loci were used for following analyses.

Number of alleles and allele frequencies were calculated using GenAlEx 6.5 (Peakall and Smouse 2012) and exact tests of Hardy Weinberg equilibrium were performed using Genepop (Rousset 2008).

\section{Population structure}

Population structure was analyzed using Bayesian clustering techniques in STRUCTURE v.2.0 (Pritchard et al. 2000). The STRUCTURE analysis was performed according to Falush et al. 2003, considering both the admixture model and the correlated allele frequencies between populations. Following recommendations by Gilbert et al. (2012), the length of the burn-in and MCMC (Monte Carlo Markov chain) were both 100,000 . The number of clusters $(K)$ was set from 
1 to 10 . For the whole dataset (170 hornets distributed between six populations), 20 runs were carried out for each value of $K$. Runs were performed twice: first, including genotypes from the 22 markers and, second, keeping genotypes from only the 15 markers developed specifically for $V$. velutina. Results from 20 runs were merged with CLUMPP (Jakobsson and Rosenberg 2007) and visualized using custom R scripts.

Genetic differentiation between populations was estimated using $F_{s t}$ calculated in ARLEQUIN v. 3.01 (Excoffier et al. 2005) and tested with 10000 random permutations of genotypes.

\section{Bottleneck detection}

We investigated the occurrence of recent genetic bottlenecks in the French population using a test of heterozygosity excess implemented in Bottleneck 1.2.0.2 (Cornuet and Luikart 1996; Piry et al. 1999). Heterozygosity excess is expected in populations that have experienced a significant reduction in size because rare alleles are lost (Cornuet and Luikart 1996). Two mutation models were tested: the infinite allele model (IAM) and the two-phase model (TPM), the latter of which incorporates elements of the IAM and stepwise mutation model $(\mathrm{SMM})($ variance $=12$, $\mathrm{SMM}=95 \%$, Piry et al. 1999). The Wilcoxon signrank test was used to test for a statistically significant bottleneck (Luikart and Cornuet 1998).

\section{Assignation test}

The program GeneClass v.2.0 (Piry et al. 2004) was used to assign or exclude reference populations (Indonesia, Vietnam, Yunnan and Zhejiang/Jiangsu) as possible origins of individuals from France and Korea on the basis of multilocus genotypes; we used the standard criterion described by Rannala and Mountain (1997). The Monte Carlo resampling method (Paetkau et al. 2004) was also applied to identify the accurate critical values of exclusion/ inclusion: our results were based on 10000 simulated genotypes for each population and a threshold probability value of 0.01 .

\section{Mating statistics}

For this study, 12 microsatellite loci were used (shown in bold and underlined in Table S2, Supporting information). Queen and mate genotypes were inferred from the worker offspring data using Colony 2.0.1.1 (Wang and Santure 2009). When available, males were also genotyped and known queen alleles were indicated in Colony 2.0.1.1 (the haploid genotypes of males are the direct product of queen meiosis). Four trials were performed with varying assumed genotyping error rates $(0.001,0.01,0.05$ and 0.1 . Allele frequencies inferred from the population study were used as input.

Inferring native range and invasion scenarios, estimating the number of foundresses and introduction date using approximate Bayesian computation

In addition to traditional population genetic approach we used the DIYABC program v.1.0.4.46 (Cornuet et al. 2008) to explore putative scenarios of invasion followed by the yellow-legged hornet in France and Korea using Approximate Bayesian Computation (ABC) method (Appendix 1 4, supplementary material). This approach was also used to estimate the effective population size of the French invasive population and the specific contribution of males and females. According to the biology of the species, founder diploid females mate with haploid males before wintering. Furthermore, in this study, mating statistics indicated that polyandry is frequent in $V$. velutina, at least in France. Finally, mitochondrial and nuclear data and multiple mating behavior suggest that, if the invasion was due to females mated before introduction, there should be mixing between one or a few female genomes but many more male genomes. This should leave a signature in the allele frequency distributions that appears bimodal. The mode at low allelic frequencies results from the less bottlenecked male founders population that partly maintained rare alleles, while the mode at high frequencies results from the more bottlenecked female population which retained only major alleles. Since the DIYABC program is able to detect admixture from frequency distribution statistics, it should detect this feature as an admixture signature between one bottlenecked population (the females) and one outbred population (the males). Accordingly, we chose to refine an invasion scenario to estimate the specific contribution of males and females. In the chosen scenario, the source of the invasive French population was a 
population close to ZHE/JIA, this population sharing more alleles with the French sample than any other native population sampled. In the refined scenario, the French invasive population (Pinv) was founded by admixture between two sources artificially modeled as independent populations, corresponding to the founder females (Pfm) and their male mates $(\mathrm{Pm})$, with different sizes ( $\mathrm{Nfm}$ and $\mathrm{Nm}$, respectively). As the male contribution was intimately associated with the female one (spermatozoids are carried by females in their spermatheca), admixture was expected to occur immediately at the foundation of the invasive population with following rates: $2 / 3$ for diploid females and $1 / 3$ for haploid males, corresponding to the expected contribution of each genomic source at equilibrium in the invasive population. In the supplementary file 1 is given the $\mathrm{R}$ code calculating the female contribution at each generation. In this scenario, only ZHE/JIA and the invasive population from France were considered. Prior settings for parameters and summary statistics were the same as for scenario comparison (see Appendix 3 and 4, Supporting information). The model was named the "sexual admixture model".

\section{Results}

Among native populations, only eight individuals could be obtained from Vietnam (VIE). This sample was however maintained in the analyses to increase the coverage of hornet native range. Some bias in allelic diversity may have arisen from this small sample size, so results from the VIE population are therefore only mentioned and not thoroughly discussed.

\section{Mitochondrial DNA analysis}

Mitochondrial haplotypes were characterized for four native regions (Zhejiang/Jiangsu, Yunnan, Vietnam and Indonesia) and two invaded regions (Korea and France), as well as for the closely related species Vespa bicolor, which was used as an outgroup. The alignment yielded a $658 \mathrm{bp}$ fragment with 120 $(18.24 \%)$ variable sites, among which 42 were parsimony-informative. Eleven COI haplotypes were detected from a total of $96 \mathrm{~V}$. velutina individuals sampled in native and invaded regions (Fig. 1; Fig. S1, Supporting information). All 11 haplotypes were found in the native area but only two of them in the introduced areas (Fig. 1), making haplotype diversity significantly higher in the native range. Furthermore, each native population showed at least two haplotypes, with haplotype diversity ranging from 0.216 to 1 and nucleotide gene diversity ranging from 0.001 to 0.038 , while both indices were 0 in the invaded areas. All sequences in France conformed to a single haplotype $\mathrm{F}$ and all those in Korea to another single haplotype, $\mathrm{K}$ (Fig. 1).

Both haplotypes $\mathrm{F}$ and $\mathrm{K}$ were also found in the native population of Zhejiang/Jiangsu but not in the other sampled regions (Yunnan, Vietnam and Indonesia), suggesting that the invasive populations are closer to this native population than to the other sampled native populations.

\section{Microsatellite diversity}

All 22 microsatellite loci analyzed in this study were polymorphic in at least one population. In France, only one locus was monomorphic (D2-142) (Tables S2 and S3, Supporting information).

No linkage disequilibrium was observed for any pair of loci after correction for multiple testing. The subsequent analyses were therefore performed on multi-locus data from all twenty-two microsatellites. Two regions (Korea and eastern China) showed no departure from Hardy Weinberg equilibrium (HWE) at any of their loci. In the four other populations, significant deviations were observed at atleast one locus. However, the departure was not systematically observed over all the loci in one population or over all the populations at one locus (Table S3). Two hypotheses may be invoked to explain these departures: the presence of population-specific null alleles and the occurrence of substructuration within populations. The latter explanation is likely, as departure from HWE was observed in populations sampled over large distances that may be subject to Walhund effect (France, Yunnan and especially Indonesia).

The mean number of alleles detected per locus per population varied from 2.7 in Korea to 7.3 in Yunnan (Table S3). Mean number of alleles per locus and expected heterozygosity were significantly higher in the native range than in introduced regions ( $P$ value of Wilcoxon's sign rank test comparing France or Korea on one side and Zhejiang/Jiangsu, Yunnan or Indonesia on the other were below 0.05 for both indices after 
correction for multiple testing, Benjamini and Yekutieli 2001).

\section{Bottleneck detection}

The decrease in genetic diversity observed in the invasive populations may be indicative of a bottleneck event. The heterozygosity excess indicating a genetic bottleneck was tested only on the French population, the sample size of Korea being too small. The result was consistent with a bottleneck: heterozygosity excess was significant $(P<0.05)$ under both IAM and TPM models.

Furthermore we visualized mode-shifts in the distribution of allele frequencies using the graphical method described in Luikart et al. (1998). Populations with stable size are characterized by an L-shaped distribution, indicative of many alleles with low frequencies. Bottlenecked populations are supposed to show a mode-shift to more alleles at intermediate frequencies, resulting from the elimination of rare alleles by founder effect. The allelic distribution in French and Korean populations revealed skewed distributions with a bimodal tendency and a "wave" at high allele frequencies (Fig. 2) indicative of bottleneck events according to Luikart et al. (1998) method.

Population structure and relationships

There was considerable divergence among populations: pairwise $F_{s t}$ ranged from 0.078 to 0.365 and all values were significantly different from zero (Table 1). Generally, $F_{s t}$ values were high between native and invasive populations (except between Korea and Zhejiang/Jiangsu) and always slightly higher between French and native populations than between Korea and native populations. For both invasive populations, the lowest $F_{s t}$ estimate was found with the Zhejiang/Jiangsu population. The $F_{s t}$ value between French and Korean invasive populations (0.33) was larger than the values between each of them and the Zhejiang/Jiangsu population ( 0.21 and
Fig. 2 Histograms of allele frequencies over all microsatellite loci for each of the invasive (FRA, KOR) and native (IND, VIE, YUN, ZHE/JIA) populations studied. Sample size (Na) is the total number of alleles. Bottlenecks are expected to shift the mode from low to high frequencies (i.e. from left to right)
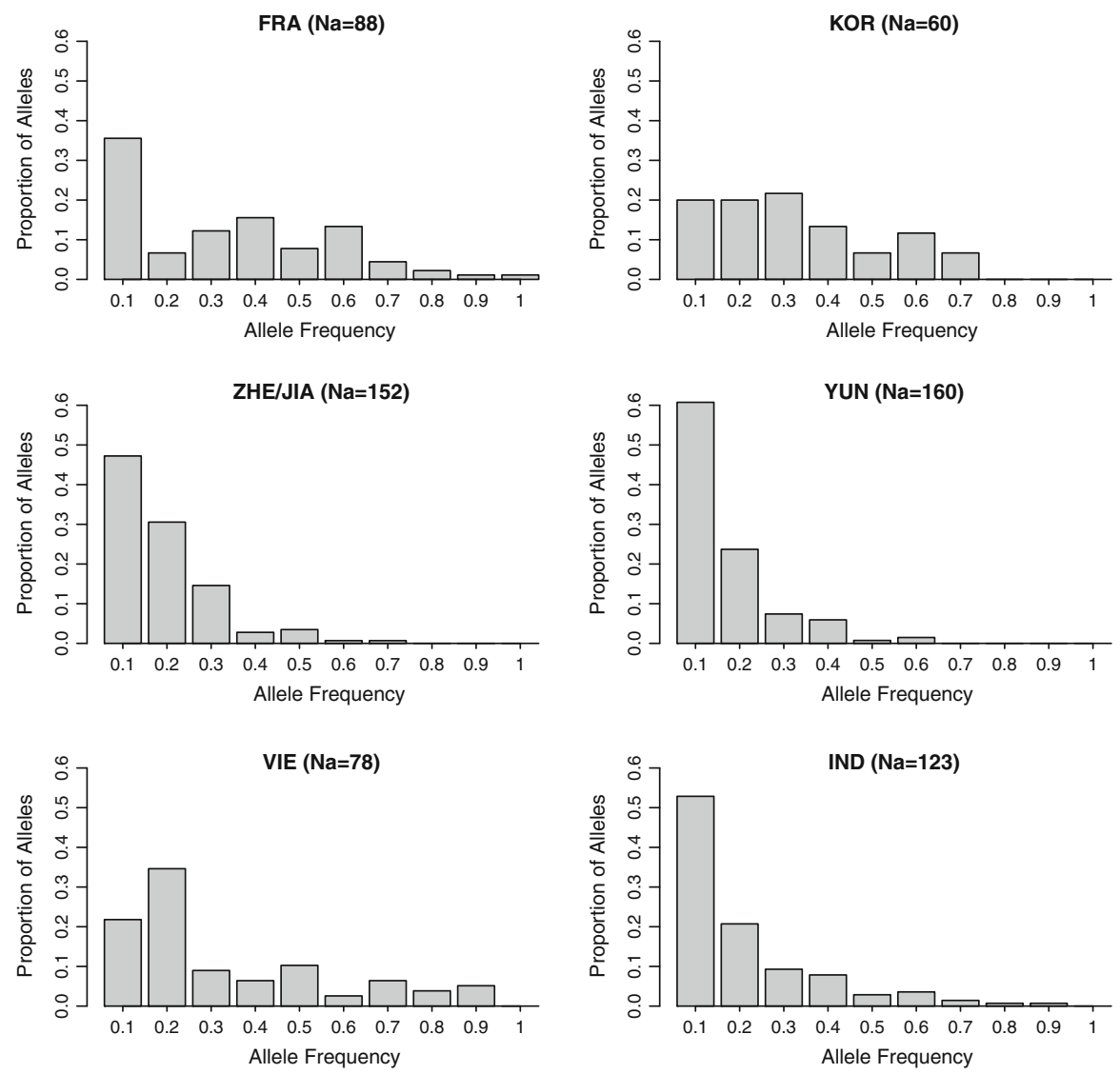
Table 1 Pairwise genetic differentiation for two introduced and four native populations of Vespa velutina

\begin{tabular}{|c|c|c|c|c|c|c|}
\hline & FRA & KOR & ZHE/JIA & YUN & VIE & IND \\
\hline FRA & 0 & & 0.7368 & 0.0112 & 0.0000 & 0.0005 \\
\hline KOR & $0.3298^{*}$ & 0 & 0.5702 & 0.0027 & 0.0000 & 0.0000 \\
\hline ZHE & $0.2087 *$ & $0.0887 *$ & 0 & & & \\
\hline YUN & $0.2274 *$ & $0.1620^{*}$ & $0.0784 *$ & 0 & & \\
\hline VIE & $0.3649 *$ & $0.3233^{*}$ & $0.2121 *$ & $0.1937 *$ & 0 & \\
\hline IND & $0.3244 *$ & $0.2385^{*}$ & $0.1534 *$ & $0.1481 *$ & $0.3053^{*}$ & 0 \\
\hline
\end{tabular}

$F_{s t}$ are indicated below the diagonal. Statistically significant values are indicated with an asterisk. The mean log assignment likelihood ( $\log L)$ of individuals from two invasive populations (France and Korea) to the four native populations are indicated above the diagonal (calculated by GeneClass 2.0. Piry et al. 2004). Invasive populations are shown in bold type

0.09 for France and Korea, respectively), suggesting two independent introductions from the native area into France and Korea.

Progressive partitioning using STRUCTURE is visualized in Fig. 3, with presentation of successive subdivisions obtained when increasing K from 2 to 10 . Despite the long run length and high number of repeats, no clustering pattern converged, as shown by the multimodal solution for some individuals, mainly from Yunnan and Zhejiang/Jiangsu. Nevertheless, some clusters coherent with geographical origin emerged when $\mathrm{K}$ was increased. The French invasive population was the first to separate from the native populations. The Indonesian population also broke away very early (from $\mathrm{K}=3$ ) and in a stable manner, despite the extent of the sampling area. Vietnam separated clearly from the other populations at $\mathrm{K}=9$. Geographical structure was not clearly resolved in Chinese populations. However, the two Chinese populations seem separated and some sub-clusters are likely to be found in Yunnan. The Korean invasive population clustered with native populations and specifically with the sample of Zhejiang/Jiangsu until $\mathrm{K}=9$. Above this value, Korean individuals clustered apart. Similar clustering emerged when the analysis was performed using only the 15 markers developed from $V$. velutina (Figure S2, Supplementary materials).

Invasive $V$. velutina sampled in France and Korea were mostly assigned by GENECLASS to the eastern Chinese province of Zhejiang/Jiangsu (Table 1). Individuals from these two invasive populations were rarely if ever assigned to the populations from Indonesia, Vietnam and Yunnan.
Genetic composition of colonies

Each of the sampled colonies had worker offspring from a single queen (Table 2). Depending on the assumed genotyping error rate, the total estimated number of patrilines varied from 22 (error rate $=0.05$ ) to 37 (error rate $=0.001$ ) over the 9 colonies studied. Even with the highly permissive $10 \%$ error rate, 7 out of the 9 queens appeared polyandrous. Furthermore, the true error rate was most probably low due to double analysis of suspect genotypes (for example unique genotypes within a queen progeny).

The mean number of matings observed per queen was between 2.44 and 4.11 and patrilines may have reached 8 in nest V0926. This result demonstrated that multiple mating is the rule in $V$. velutina at least in France (Table 3).

Number of founders and introduction date

The number of introduced foundresses, their number of mates and the date of the first introduction were inferred under the sexual admixture model using the DIYABC program. Considering the high genetic proximity found between Zhejiang/Jiangsu population and France (also supported by DIYABC study, Appendix 5, Figure S3 and Table S4 and S5, Supplementary material), this native population was considered as the invasion source. Both the average relative bias and the RMSE were low and did not indicate any systematic over- or underestimation of the various parameters. None of the estimated parameters, except Ninv, had bias or RMSE higher than 


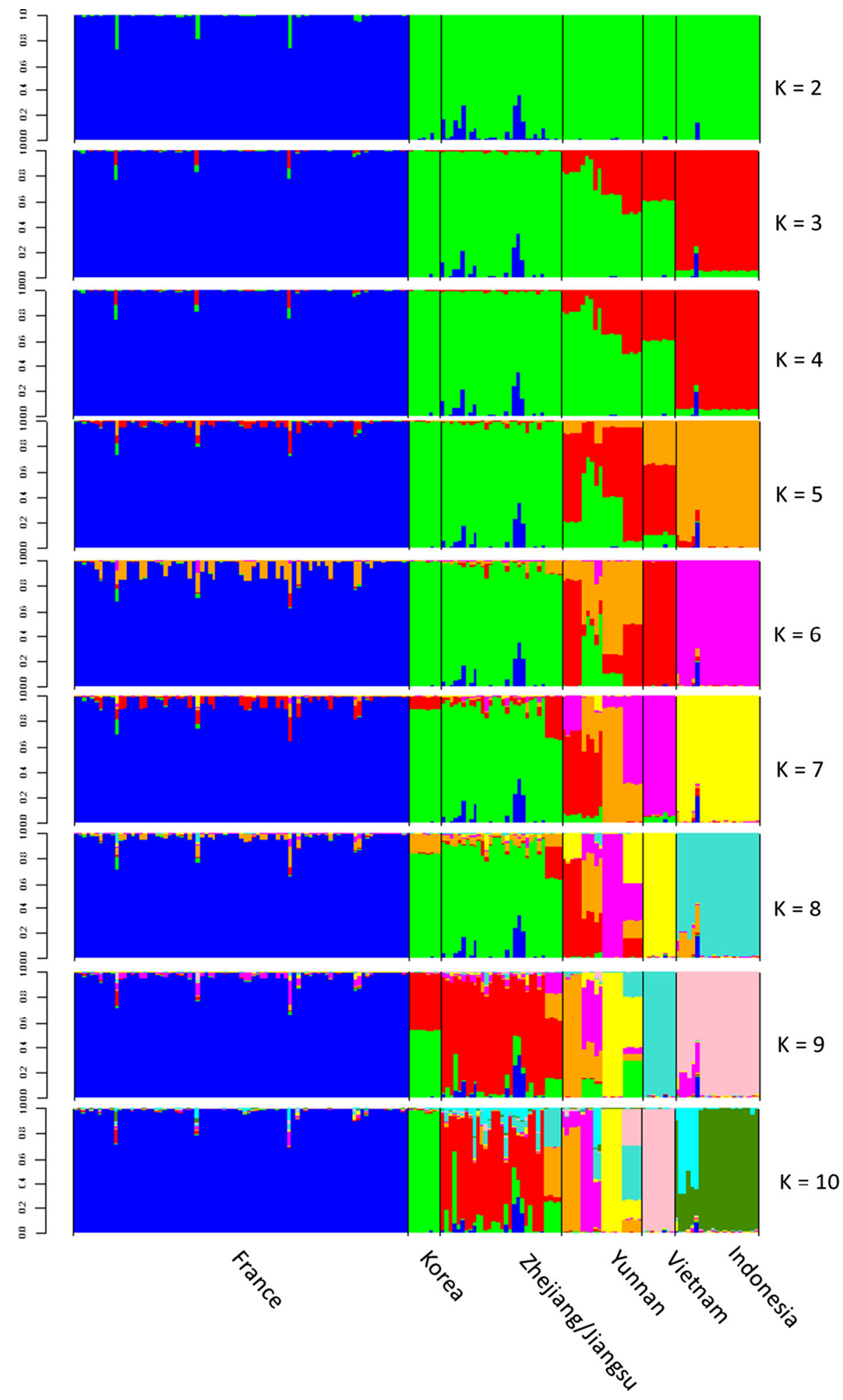

Fig. 3 Graphical output from STRUCTURE (Pritchard et al. 2000) for each value of $\mathrm{K}$ from 1 to 10 [modified in DISTRUCT (Rosenberg et al. 2002)]. Each vertical line represents an individual, and the color composition displays the probability of belonging to each of the 210 clusters defined by STRUCTURE 
Table 2 Number of queens and mates per colony in the French population, inferred from the worker offspring data using Colony 2.0.1.1

\begin{tabular}{lrlclcl}
\hline & M n & F 2n & NLoc & Nq & Nm \\
\hline V0809 & 0 & 51 & 10 & 1 & 13 \\
V0813 & 10 & 48 & 8 & 1 & 3 & 5 \\
V0825b & 25 & 42 & 11 & 1 & 13 \\
V0830 & 0 & 41 & 12 & 1 & 3 & 6 \\
V0907 & 20 & 43 & 12 & 1 & 2 & 5 \\
V0912 & 0 & 13 & 8 & 1 & 23 \\
V0921 & 0 & 45 & 8 & 1 & 12 \\
V0926 & 5 & 76 & 11 & 1 & 5 & 8 \\
V0927 & 0 & 36 & 12 & 1 & 2 & 4 \\
All nests & & & & & 2.44 & 4.11 \\
\hline
\end{tabular}

$M n$ number of analyzed males per colony, $F 2 n$ number of analyzed females per colony, NLoc number of analyzed loci per individual, $N q$. number of queens per colony, $N m$ number of mates per queen (range variation is given between the four analyses with different genotyping error rates)

one. In general, the width of the $50 \%$ credible intervals was small, showing that the parameters were reasonably well estimated; all values of the parameter estimates resulting from the pseudo-observed data sets lie between 50 and $200 \%$ of the estimated median values. The lack of confidence in the estimate of present effective size of the French population may have resulted from the expanding status of this population, as the hornet is still colonizing new territories in France and Europe. Nevertheless, confidence was far larger for other parameters. From this sexual admixture model, we estimated the number of introduced females as only 1 (0.99) and its number of mates as 1.8 diploid males, corresponding to 3.6 haploid males. The time of introduction into France (ti) was estimated at 7.48 generations, corresponding to between 7 and 8 years before sampling.

\section{Discussion}

The primary objective of this study was to genetically characterize the introduction events of Vespa velutina in Europe and Korea in order to infer the number of introduced populations, their origins and their sizes. Results are more reliable for France, as sampling effort was larger for this invaded territory than for Korea, though general conclusions may apply to both invasion histories. A recent study that used larger sample sizes from Korean populations confirms these general conclusions (Choi et al. 2013).

Geographical origin of invaders

Several studies have shown that identifying a source population precisely can be challenging if the potential source region is large, genetically homogeneous or not differentiated (Wares et al. 2005). In our study, genetic differentiation between the native populations was high providing theoretically optimal conditions for accurate source determination (Guillemaud et al. 2011). When differentiation between populations is high, a large sampling effort may be required to ensure that the real source population is not missed. We only obtained sparse samples from the native range of the species and the precise geographic origin of invasive population has to be confirmed. Nonetheless, our results strongly suggest that the origin of the Korean and the French invasive populations of $V$. velutina originated from an eastern Chinese population. The two mitochondrial haplotypes detected in each invaded area to date were also found in two neighboring localities (37 km apart) in the two Chinese provinces of Jiangsu and Zhejiang. Furthermore, analysis of nuclear markers indicates that the recently established French and Korean populations are more closely related to the eastern Chinese population than to the other Asian populations. These findings were also in agreement with the assignment test conducted with GeneClass. The high percentage of individuals from the invaded areas assigned to the Zhejiang/Jiangsu population and the high significance of these assignments suggest that the more likely source for introduction among the available samples is this eastern Chinese coastal area. Approximate Bayesian Computation also suggested a population source closely related to Zhejiang/Jiangsu. The inferred eastern China origin of the French invasive population analyzed here is consistent with factual information about the commercial exchanges that were probably responsible for the introduction of $V$. velutina into France. Such an event is also in agreement with the increased importance of Asia as a source of alien hymenopteran species in Europe (Rasplus et al. 2010). Nevertheless we have to take into account the fact that we only obtained sparse samples from the native range of the species. A larger sampling effort may be 
Table 3 Prior settings, parameter estimations (median of the posterior distribution) and confidence in these estimations for the scenario devoted to inferring the number of introduced individuals and the date of the first introduction in France

\begin{tabular}{|c|c|c|c|c|c|c|c|c|}
\hline Parameter & Priors & Estimate & & Bias & RMSE & $50 \% \mathrm{cov}$ & $95 \% \mathrm{cov}$ & Fact 2 \\
\hline Ns & $\mathrm{U}\left[\begin{array}{ll}1 & 10000\end{array}\right]$ & [median & $\left.3.2 \times 10^{3}\right]$ & 0.217 & 0.894 & 0.532 & 0.962 & 0.878 \\
\hline$t 1$ & $\mathrm{U}\left[\begin{array}{ll}1 & 10\end{array}\right]$ & [median & 7.48] & 0.236 & 0.976 & 0.554 & 0.954 & 0.854 \\
\hline Ninv & $\mathrm{U}\left[\begin{array}{ll}1 & 10000\end{array}\right]$ & [median & $\left.9.38 \times 10^{2}\right]$ & 2.57 & 18.237 & 0.512 & 0.948 & 0.716 \\
\hline $\mathrm{Nm}$ & $\mathrm{U}\left[\begin{array}{ll}1 & 20\end{array}\right]$ & [median & $1.80]$ & 0.021 & 0.318 & 0.538 & 0.962 & 0.988 \\
\hline$N f m$ & $\mathrm{U}\left[\begin{array}{ll}1 & 20\end{array}\right]$ & [median & $\left.9.90 \times 10^{-1}\right]$ & 0.084 & 0.38 & 0.506 & 0.938 & 0.986 \\
\hline
\end{tabular}

The validity of the parameter estimates was evaluated with DIYABC by comparing the estimates obtained from the ABC analysis of 100 pseudo observed datasets (pods) simulated from parameter values drawn from the prior distributions and scenario 1 (Appendix 14 , Table S5 supplementary material) with the true value of the parameters (i.e. the values drawn from the priors). Then the relative mean square error (RMSE: the square root of the average square difference between the $100 \mathrm{ABC}$ estimates and the actual parameter value) and the relative bias (the mean absolute difference between the $100 \mathrm{ABC}$ estimates and the actual parameter value divided by the actual parameter value), the 90 and the $50 \%$ coverage (i.e. the fraction of 90 and $50 \%$ highest posterior densities obtained from the $100 \mathrm{ABC}$ analyses that includes the actual parameter value), and the factor 2 (the proportion of the $100 \mathrm{ABC}$ estimates obtained that are within the range of $X / 2$ and $2 X$, with $X$ the actual parameter value)

$N s$ Zhejiang/Jiangsu effective population size. $t 1$ time of introduction to France. Ninv French effective population size. $N m$ effective number of haploid males that mated with introduced founders. $\mathrm{Nfm}$ effective number of introduced foundresses. Note that the estimate of $\mathrm{Nfm}$ is outside the prior distribution range. This an artifact due to the linear regression used in the $\mathrm{ABC}$ procedure. The estimate is therefore considered to be one in the following. Strongly biased and/or imprecise estimates are highlighted in bold. U $[\mathrm{X}, \mathrm{Y}]$ refers to as a uniform distribution with $\mathrm{X}$ and $\mathrm{Y}$ as lower and upper limits, respectively

required to ensure that the real source population has not been missed.

Estimated number of introduced individuals

A notable characteristic of the invasive hornet populations in France and Korea is their impoverished genetic diversity compared with native populations from Asia. A single mtDNA haplotype was found in each of these invasive populations, while 23 haplotypes were sampled in populations from the native range. Genetic diversity at microsatellite loci was also lower in all invasive populations than in native populations, with less than half the allelic richness, and lower levels of heterozygosity. Furthermore, native populations were well differentiated from each other, with even some substructuration observed in the Yunnan area. In contrast, the French population appeared as a single homogeneous genetic group for any number of clusters tested. Choi et al. (2013) studied larger samples from two localities in Korea (44 and 43 samples, respectively) with 7 of the microsatellite markers included in the present study. Their results are in complete agreement with the results we obtained: (1) they showed a clear depletion of genetic diversity in Korea compared with native populations,
(2) they found evidence of a genetic bottleneck when testing with BOTTLENECK software, and (3) they did not detect any genetic structure in the Korean population.

Taken together, highly reduced levels of genetic variation in introduced $V$. velutina populations along with significant bottlenecks and the lack of related population structure suggest that this hornet experienced a single and severe founder event in both Korea and France.

Several aspects of the data suggested that the strength of the bottleneck in Europe arose from the introduction of very few or possibly a single foundress. This hypothesis is supported by the presence of a single mitochondrial haplotype in France. Indeed, each colony is generally founded by a single diploid female, resulting in the presence of a unique mtDNA in the colony and future queens. Results associated with nuclear markers are, however, not compatible with the introduction of a singly-mated female for a number of reasons. First, more than three alleles are observed at many loci in France. Second, the drop-off in diversity is more pronounced in mitochondrial DNA than in nuclear DNA contrarily to what is described in other species that have experienced a similar invasion pattern (Mikheyev 
et al. 2009). Combined mitochondrial and nuclear data suggest that the establishment of the present population of yellow-legged hornet in France would have been made possible by the introduction of very few or, as suggested by the DIYABC analysis, a single queen fertilized by several males. The discovery of frequent polyandry in Vespa velutina favors this scenario.

We used an ABC approach to test the hypothesis that the invasion of France originated from the introduction of a small number of founder females fertilized by several males. The ABC analyses confirmed that all of the French hornet samples were likely derived from a single female, and the effective number of their haploid male mates $(\mathrm{Pm})$ was estimated at $3.6\left(\mathrm{Cl}=\left[\begin{array}{ll}2.00 & 17.26\end{array}\right]\right)$. This result is consistent with the independent 'Colony' analysis from which we estimated that females in France are fertilized by 2.444 .11 males. A precise estimation could not be obtained for the invasive Korean population, due to small sample size.

Genetic bottlenecks and loss of substantial amount of genetic diversity are common features of invasive populations (Puillandre et al. 2008; Dlugosch and Parker 2008). Another famous case of successful invasion with a large decrease in diversity in the introduced range concerns Bombus terrestris, the European bumblebee. In Tasmania, for example, an invasion was apparently caused by the introduction of a very small number of individuals from a single source region, perhaps as few as two bees (SchmidHempel et al. 2007). The high success of some species, despite such large genetic losses is a well-known paradox (Dlugosch and Parker 2008).

Invasive success despite a small propagule size

Several studies on conservation genetics have demonstrated that reduced genetic variation due to genetic drift following a founder effect limits the ability of a population to adapt, and small population size increases the risk of extinction (Frankham and Ralls 1998; Allendorf and Lundquist 2003). Particularly in isolated haplodiploid populations, where sex is often determined by a single locus, increased homozygosity due to inbreeding increases the production of nonviable or effectively sterile diploid males, which reduces population growth rates and effective sizes, thus potentially creating a rapid extinction vortex (Zayed et al. 2007; Dlugosch and Parker 2008). The invasion of the yellow-legged hornet in France is an exemplary case of invasive success from a very small propagule size. Paradoxically, V. velutina appears to be able to establish even after a severe genetic bottleneck resulting from the introduction of very few or possibly a single female (although multiply mated). Furthermore, the species has succeeded in two apparently independent cases of introduction: in Europe and in Korea. Several species of ant and wasp have become highly invasive in their introduced range, but have generally not experienced reductions in propagule pressure on the same scale as $V$. velutina (Tsutsui and Suarez 2003). A rare example is the case of the little fire ant Wasmannia auropunctata, for which there is evidence that only a single female or a small group of sisters was introduced to Central Africa and Hawaii. The unusual, largely clonal, reproductive strategy of $W$. auropunctata may have enhanced its success as an invasive species (Mikheyev et al. 2009).

Admittedly, many biological and environmental factors might have contributed to the invasiveness of $V$. velutina in France and counterbalanced the negative effect on genetic diversity of small number of female foundresses; these might include the suitable climatic conditions, the abundance of honeybees and, according to Villemant et al. (2011), the low level of competition from other Vespa sp. that $V$. velutina faces in Europe. Additionally, our results also point to the contribution that reproductive system traits of this species may have made to the success of the invasion, by increasing the number of male founders.

In this paper, we demonstrated that $V$. velutina is a polyandrous species. According to a recent data compilation (Hughes et al. 2008), V. velutina is the only Vespa species to date to present moderate polyandry (defined by the authors as 210 effective mates), whereas $V$. crabro and $V$. mandarina exhibit facultative low polyandry and $V$. ducalis is monoandrous. Available data highlight the fact that $V$. velutina queens exhibit one of the highest mean effective mating frequencies among the Vespidae. Interestingly, among the few other Vespidae species showing moderate polyandry, Vespula germanica and Vespula vulgaris also revealed to be invasive species (Beggs et al. 2011). Similarly, the successful invasion of leaf-cutting ant Acromyrmex octospinosus in Guadeloupe is likely the result of a multimated female (Mikheyev 2008). Despite the cost of multiple mating, polyandry could be beneficial to colonies, not 
only because monogynous queens need sperm from several males in order to produce enough workers, but also because it may offer several other genetic advantages that may have determined the success of invasion (Crozier and Fjerdingstad 2001). Notably, multiple mating implies higher genetic variation among offspring (Yasui 1998), which can improve the resistance of the whole colony to parasites or pathogens (Schmid-Hempel and Crozier 1999) and increase colony survival under variable environmental conditions (Jennions and Petrie 2000); this may be of importance for adaptation to a new environment in an invaded area. Genetic diversity reduction observed in a population developed from a single female is far less severe when this female is multiply mated than when it is only singly mated. As a result, polyandry may also decrease diploid male load at the colony level by decreasing the production of diploid males resulting from the sex determination mechanism (Jennions and Petrie 2000).

\section{Conclusion}

The results of this study, based on a number of highly informative genetic markers, provide the first genetic reconstruction of the $V$. velutina invasion in Europe. The congruence between the results based on the different classes of markers and different analytical approaches adds weight to the credibility of our estimates.

The species has clearly proven its high invasive potential: our genetic study shows that the introduction of a very small number or even probably a single multi-mated queen can initiate a full-scale invasion. This discovery represents a challenge to management practices intended to control such introductions or to remove them from non-native environments. Rapid detection at the port of entry seems to be the best way to prevent a large scale infestation, although just one escaped founder, or a very small number, can render management actions ineffective.

It is possible that other invasive species share these characteristics and could become invaders even if only a very small number of individuals are introduced. It is therefore a priority to distinguish these species so that they can be managed with particular caution, as traditional practices may be insufficient for their control.
Acknowledgments We thank Daniel Simberloff for his very useful rereading of an earlier version of the manuscript. We thank all the French beekeepers and associations that kindly provided us with Asian yellow legged hornet samples from France. We also thank our colleagues Alain Roques (INRA, Orléans), Agnès Rortais (CNRS, Gif sur Yvette), Pierre Tripotin (MNHN, Paris), Tan Ken (Chinese Academy of Sciences, China), Yayuk Suhardjono and Oscar Effendy (Museum of Bogor, LIPI, Indonesia), and Truong Quang Tam (Institute of Tropical Biology, Vietnam) for help with sample collection from Asia. Cyril Nadeau, Delia Dupré and Ugoline Godeau provided technical help in the laboratory. This study was financially supported by France AgriMer (Programme communautaire pour l'Apiculture, 2008 2011) and IRD and CNRS core budgets. Collecting missions were supported by the MNHN for Vietnam (PPF 2008); Indonesia (ATM Biodiversité 2010), China (ATM Formes 2010). We thank Emmanuelle Baudry (UPSud, Orsay) for fruitful comments on the manuscript. The English was revised by Helen McCombie.

\section{References}

Abrol DP (1994) Ecology, behaviour and management of social wasp, Vespa velutina Smith (Hymenoptera: Vespidae), attacking honeybee colonies. Korean J Apic 9:5

Allendorf FW, Lundquist LL (2003) Introduction: population biology, evolution, and control of invasive species. Con serv Biol 17:24 30

Arca M, Capdevielle Dulac C, Villemant C et al (2011) Development of microsatellite markers for the yellow legged Asian hornet, Vespa velutina, a major threat for European bees. Conserv Genet Resour 2:283 286

Beggs JR, Brockerhoff EG, Corley JC et al (2011) Ecological effects and management of invasive alien Vespidae. Bio control 56:505 526

Benjamini Y, Yekutieli D (2001) The control of the false dis covery rate in multiple testing under dependency. Ann Statist 29(4):1165 1188

Carpenter JM, Kojima J (1997) Checklist of the species in the subfamily Vespinae (Insecta: Hymenoptera: Vespidae). Natural history bulletin of Ibaraki University 1:51 92

Chapman RE, Bourke AFG (2001) The influence of sociality on the conservation biology of social insects. Ecol Lett 4:650 662

Choi MB, Martin SJ, Lee JW (2012) Distribution, spread and impact of the invasive hornet Vespa velutina in South Korea. Asia Pacific Entomol 15:473 477

Choi MB, Lee S A, Suk HY, Lee JW (2013) Microsatellite variation in colonizing populations of yellow legged Asian hornet, Vespa velutina nigrithorax. South Korea. Entomol Res 43(4):208 214

Clement M, Posada D, Crandall KA (2000) TCS: a computer program to estimate gene genealogies. Mol Ecol 9:1657 1659

Cornuet JM, Luikart GL (1996) Description and power analysis of two tests for detecting recent population bottlenecks from allele frequency data. Genetics 144:2001 2014

Cornuet JM, Santos F, Beaumont MA et al (2008) Inferring population history with DIY ABC: a user friendly 
approach to approximate Bayesian computation. Bioin formatics 24:2713 2719

Crozier R, Fjerdingstad EJ (2001) Polyandry in social Hy menoptera disunity in diversity? Annls Zool Fennici 38:267 285

Daly D, Archer ME, Watts PC et al (2002) Polymorphic mi crosatellite loci for eusocial wasps (Hymenoptera: Ve spidae). Mol Ecol Notes 2:273

Dlugosch KM, Parker IM (2008) Founding events in species invasions: genetic variation, adaptive evolution, and the role of multiple introductions. Mol Ecol 17:431

Excoffier L, Laval G, Schneider S (2005) Arlequin (version 3.0): an integrated software package for population genetics data analysis. Evol Bioinform 1:47 50

Falush D, Stephens M, Pritchard JK (2003) Inference of popula tion structure using multilocus genotype data: linked loci and correlated allele frequencies. Genetics 164:1567 1587

Folmer O, Black M, Hoeh W, Lutz R, Vrijenhoek R (1994) DNA primers for amplification of mitochondrial cy tochrome c oxidase subunit I from diverse metazoan in vertebrates. Mol Mar Biol Biotechnol 5:294 299

Frankham R, Ralls K (1998) Conservation biology: inbreeding leads to extinction. Nature 392:441 442

Gilbert KJ, Andrew RL, Bock DG, Franklin MT, Kane NC, Moore JS, Moyers BT, Renaut S, Rennison DJ, Veen T, Vines TH (2012) Recommendations for utilizing and re porting population genetic analyses: the reproducibility of genetic clustering using the program STRUCTURE. Mol Ecol 21:4925 4930

Guillemaud T, Ciosi M, Lombaert E, Estoup A (2011) Biolo gical invasions in agricultural settings: insights from evo lutionary biology and population genetics. C R Biol 33:237 246

Hajibabaei M, deWaard JR, Ivanova NV et al (2005) Critical factors for assembling a high volume of DNA barcodes. Philos Trans R Soc Lond B Biol Sci 360:1959 1967

Hall TA (1999) BioEdit: a user friendly biological sequence alignment editor and analysis program for Windows 95/98/ NT. Nucleic Acids Symp Ser 41:95 98

Hasegawa E, Takahashi J (2002) Microsatellite loci for genetic research in the hornet Vespa mandarinia and related spe cies. Mol Ecol Notes 2:306

Holway DA, Lach L, Suarez AV, Tsutsui ND, Case TJ (2002) The causes and consequences of ant invasions. Annu Rev Ecol Evol Syst 33:181 233

Hughes WOH, Ratnieks FLW, Oldroyd BP (2008) Multiple paternity or multiple queens: two routes to greater intra colonial genetic diversity in the eusocial Hymenoptera. J EvolutionBiol 21:1090 1095

Ings TC, Ward NL, Chittka L (2006) Can commercially im ported bumble bees out compete their native conspecifics? J Appl Ecol 43:940 948

Jakobsson M, Rosenberg NA (2007) CLUMPP: a cluster matching and permutation program for dealing with label switching and multimodality in analysis of population structure. Bioinformatics 23:1801

Jennions MD, Petrie M (2000) Why do females mate multiply? A review of the genetic benefits. Biol Rev Camb Philos Soc 75:21 64

Ken T, Hepburn HR, Radloff SE et al (2005) Heat balling wasps by honeybees. Naturwissenschaften 92:492 495
Luikart G, Allendorf F, Cornuet JM, Sherwin W (1998) Dis tortion of allele frequency distributions provides a test for recent population bottlenecks. J Hered 89:238

Luikart G, Cornuet JM (1998) Empirical evaluation of a test for identifying recently bottlenecked populations from allele frequency data. Cons Biol 12(1):228 237

Mack RN, Simberloff D, Lonsdale WM et al (2000) Biotic in vasions: causes, epidemiology, global consequences, and control. Ecol Appls 10:689 710

Mikheyev AS (2008) History, genetics and pathology of a leaf cutting ant introduction: a case study of the Guadeloupe invasion. Biol Invasions 10:467 473

Mikheyev AS, Bresson S, Conant P (2009) Single queen in troductions characterize regional and local invasions by the facultatively clonal little fire ant Wasmannia auropunctata. Mol Ecol 18:2937 2944

Moller H (1996) Lessons for invasion theory from social insects. Biol Cons 78:125 142

Monceau K, Arca M, Leprêtre L, Mougel F, Bonnard O, Silvain JF, Maher N, Arnold G, Thiéry D (2013) Native prey and invasive predator patterns of foraging activity: the case of the yellow legged hornet predation at European honeybee hives. PLoS ONE 8(6):e66492

Nei M (1987) Molecular evolutionary genetics. Columbia University Press, New York

Paetkau D, Slade R, Burden M, Estoup A (2004) Genetic as signment methods for the direct, real time estimation of migration rate: a simulation based exploration of accuracy and power. Mol Ecol 13:55 65

Peakall R, Smouse PE (2012) GenAlEx 6.5: genetic analysis in excel. Population genetic software for teaching and re search an update. Bioinformatics 28:2537 2539

Perrard A, Haxaire J, Rortais A, Villemant C (2009) Observa tions on the colony activity of the Asian hornet Vespa ve lutina Lepeletier 1836 (Hymenoptera: Vespidae: Vespinae) in France. Ann Soc Entomol Fr 45:119 127

Piry S, Luikart G, Cornuet JM (1999) Bottleneck: a computer program for detecting recent reductions in the effective population size using allele frequency data. J Hered 90:502 503

Piry S, Alapetite A, Cornuet JM et al (2004) GENECLASS 2: a software for genetic assignment and first generation $\mathrm{mi}$ grant detection. J Hered 95:536

Pritchard JK, Stephens M, Donnelly P (2000) Inference of population structure using multilocus genotype data. Ge netics 155:945 959

Puillandre N, Dupas S, Dangles O et al (2008) Genetic bottle neck in invasive species: the potato tuber moth adds to the list. Biol Invasions 10:319 333

Rannala B, Mountain JL (1997) Detecting immigration by using multilocus genotypes. Proc Natl Acad Sci USA (PNAS) 94:9197 9201

Rasplus JY, Villemant C, Paiva MR, Delvare G, Roques A (2010) Hymenoptera. In: Roques A, Kenis M, Lees D (eds) Arthropod invasions in Europe. BioRisk 4. Pensoft, Sofia, pp 669776

Rome Q, Dambrine L, Onate C, Muller F, Villemant C, Garcia Perez L, Maia M, Carvalho Esteves P, Bruneau E (2013) Spread of the invasive hornet Vespa velutina Lepeletier, 1836, in Europe in 2012 (Hym., Vespidae). Bull Soc en tomol Fr 118:21 22 
Rortais A, Villemant C, Gargominy O, Rome Q, Haxaire J, Papachristoforou A, Arnold G (2010) A new enemy of honeybees in Europe: the Asian hornet Vespa velutina. In: Settele J (ed) Atlas of biodiversity risks from Europe to the globe, from stories to maps. Pensoft, Sofia

Rosenberg NA, Pritchard JK, Weber JL, Cann HM, Kidd KK, Zhivotovsky LA, Feldman MW (2002) Genetic structure of human populations. Science 298:2381 2385

Rousset F (2008) Genepop'007: a complete reimplementation of the Genepop software for Windows and Linux. Mol Ecol Resources 8:103 106

Rozas J, Sánchez DelBarrio JC, Messeguer X, Rozas R (2003) DnaSP, DNA polymorphism analyses by the coalescent and other methods. Bioinformatics 19:2496 2497

Schmid Hempel P, Crozier RH (1999) Polyandry versus poly gyny versus parasites. Philos Trans R Soc B Biol Sci 354: 507515

Schmid Hempel P, Schmid Hempel R, Brunner PC, Seeman OD, Allen GR (2007) Invasion success of the bumblebee, Bombus terrestris, despite a drastic genetic bottleneck. Heredity 99:414 422

Suarez AV, Tsutsui ND (2008) The evolutionary consequences of biological invasions. Mol Ecol 17:351 360

Tan K, Radloff S, Li JJ, Hepburn HR, Yang MX, Zhang LJ, Neumann P (2007) Bee hawking by the wasp, Vespa ve lutina, on the honeybees Apis cerana and A. mellifera. Naturwissenschaften 94:469 472

Templeton AR, Crandall KA, Sing CF (1992) A cladistics ana lysis of phenotypic associations with haplotypes inferred from restriction endonuclease mapping and DNA sequence data, III. Cladogram estimation. Genetics 132:619 633
Tsutsui ND, Suarez AV (2003) The colony structure and population biology of invasive ants. Conserv Biol 17:48 58

Tsutsui ND, Suarez AV, Holway DA, Case TJ (2001) Rela tionships among native and introduced populations of the Argentine ant (Linepithema humile) and the source of in troduced populations. Mol Ecol 10:2151 2161

Villemant C (2008) Apis cerana se défend contre Vespa velutina : observations dans le massif forestier du Bi Doup, Viet nam. Bull Soc entomol Fr 113:312

Villemant C, Haxaire J, Streito JC (2006) Premier bilan de l'invasion de Vespa velutina Lepeletier en France (Hy menoptera, Vespidae). Bull Soc entomol Fr 111:535

Villemant C, Barbet Massin M, Perrard A et al (2011) Pre dicting the invasion risk by the alien bee hawking yellow legged hornet Vespa velutina nigrithorax across Europe and other continents with niche models. Biol Cons $144: 21422150$

Wang J, Santure AW (2009) Parentage and sibship inference from multilocus genotype data under polygamy. Genetics 181(4):1579 1594

Wares JP, Hughes AR, Grosberg RK (2005) Mechanisms that drive evolutionary change: insights from species introduc tions and invasions. In: Sax DF, Stachowicz JJ, Gaines SD (eds) Species invasions: insights into ecology, evolution and biogeography. Sinauer, Sunderland, MA, pp 229257

Sinauer, Sunderland, MA. Yasui Y (1998) The "genetic benefits" of female multiple mating reconsidered. Trends Ecol Evol 13:246 250

Zayed A, Constantin SA, Packer L (2007) Successful biological invasion despite a severe genetic load. PLoS ONE 2(9):e868 